\title{
Schwanoma múltiple del pene
}

\author{
Simeão Versos R, Louro N, Borges R, Palacios A, Silva Ramos M, Queiróz J , Marcelo F. \\ Servicio de Urología. Hospital General de Santo Antonio. Porto, Portugal.
}

Actas Urol Esp. 2006;30(8):824-828

\section{RESUMEN}

\section{SCHWANOMA MÚLTIPLE DEL PENE}

El schwanoma o neurilemoma es un tumor con origen en las células de Schwann de la vaina de los nervios periféricos. En la literatura están descritos veintisiete casos de schwanomas del pene.

Presentamos un caso de schwanoma múltiple del pene, haciendo una breve revisión de la literatura sobre este raro tumor con respecto a su etiopatogenia, diagnóstico, tratamiento y orientación.

Palabras clave: Schwanoma. Neurilemoma. Pene. Cuerpos de Verocay. Proteína S-100.

\section{ABSTRACT \\ MULTIPLE SCHWANNOMA OF THE PENIS}

Schwannoma or neurilemmoma designate a tumour that originates in the Schwann cells of peripheral nerves, therefore occurring anywhere in the body. They are very rare in the penis. In the literature are reported twenty seven cases of penile schwannoma.

We report a case of multiple schwannoma of the penis and make a brief literature revision about this rare pathology.

Keywords: Schwannoma. Neurilemmoma. Penis. Verocay bodies. S-100 protein.

$\mathrm{E}^{2}$ schwanoma es un tumor raro que se desarrolla a partir de las células de Schwan de las vainas de los nervios periféricos, por lo que puede aparecer en cualquier parte del organismo. Aparece frecuentemente en la cabeza, cuello y extremidades ${ }^{1}$ y no se encuentran factores predisponentes para su formación. En el aparato genitourinario está descrito en el retroperitoneo, cordón espermático, testículo, túnica vaginal testicular, pene, vesículas seminales y próstata ${ }^{2,3}$.

En la mayoría de los casos son lesiones benignas, únicas y de crecimiento lento. El tratamiento es quirúrgico y el diagnóstico depende del examen histológico con la identificación de los cuerpos de Verocay e inmunocitoquímico con la identificación de la proteína S-100.

Las lesiones malignas casi siempre están asociadas a neurofibromatosis tipo I o enfermedad de Von Recklinghausen ${ }^{4}$.

\section{CASO CLÍNICO}

Hombre de 58 años, caucasiano, quien consulta por múltiples lesiones nodulares en el lado derecho de la base del pene, indoloras, que no provocaban disfunción sexual, síntomas miccionales, ni curvatura peneana, y con más de 3 años de evolución.

$\mathrm{Al}$ examen físico se palpaban múltiples (3-4) lesiones nodulares, de aspecto ovoide, de volumen variable e inferior a $3 \mathrm{~cm}$, de consistencia duro-elástica, localizadas en el lado derecho de la base peneana (Fig. 1), sin adherencia a los planos superficiales. No se detectan induraciones ni manchas cutáneas en otras localizaciones.

Analíticamente presentaba colesterol total de 257 y triglicéridos dentro de la normalidad.

La ecografía (Fig. 2) revelaba 3 formaciones quísticas de 11,12 y $17 \mathrm{~mm}$ de diámetro, $2 \mathrm{de}$ contenido anecoico y una con ecos en su interior, 


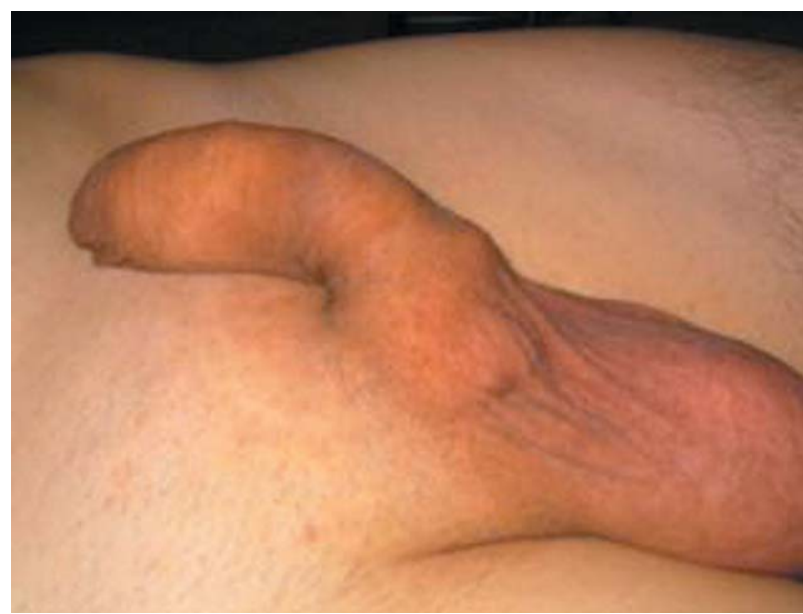

FIGURA 1. Lesiones nodulares en la base del pene.

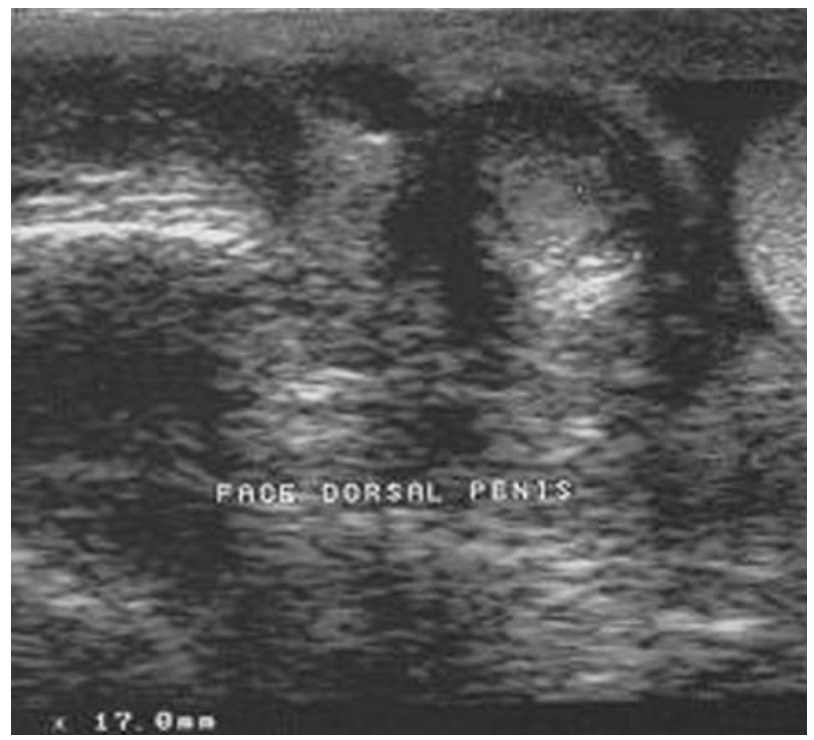

FIGURA 2. Ecografia: lesiones quisticas subcutáneas ovales de dimensión variable.

superficiales, localizadas en la vertiente derecha de los cuerpos cavernosos y sin relación con los mismos.

La resonancia magnética nuclear (RMN) (Fig. 3) mostraba 3 formaciones quísticas localizadas en la base lateral derecha de la raíz del pene sin relación con los cuerpos cavernosos.

Efectuamos la exéresis completa de 4 tumores mediante incisión para-peneana derecha (Fig. 4), constatando que se encontraban localizados por debajo de la fascia de Buck y por encima de la albuginea del cuerpo cavernoso derecho, presentándose como formaciones bien delimitadas, capsuladas y de color uniformemente pálida.

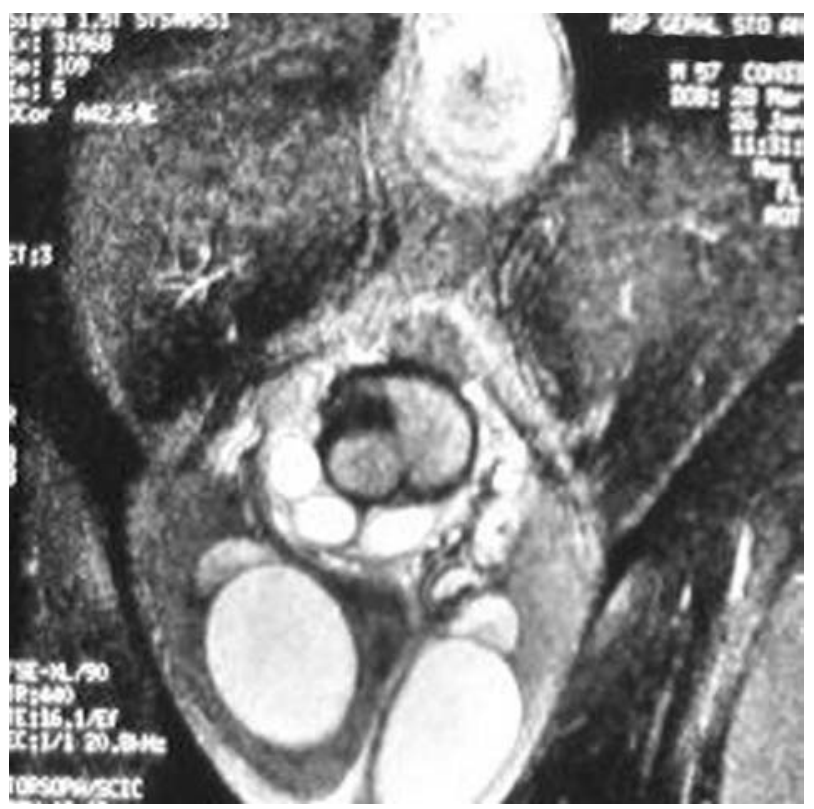

FIGURA 3. RMN: Evidencia de lesiones quisticas bien delimitadas, por encima de la albuginea cavernosa (corte coronal); hiperseñal en T2.

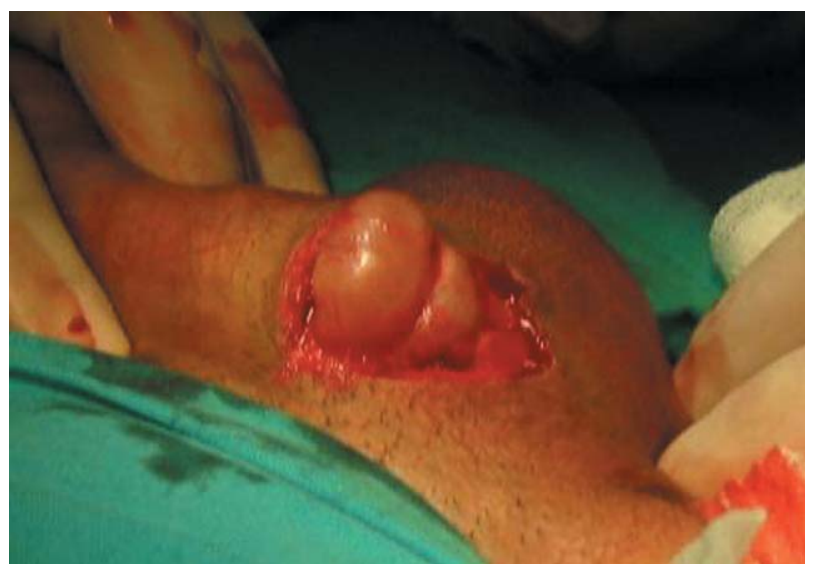

FIGURA 4. Incisión para-peneana derecha con exposición de 2 tumores.

El examen macroscópico de la pieza revelaba 4 formaciones nodulares ovaladas de $20 \times 15 \mathrm{~mm}$, $15 \times 10 \mathrm{~mm}, 6 \times 4 \mathrm{~mm}$ y $4 \mathrm{~mm}$ de diámetro, de superficie lisa y constituidos por tejidos firmes, blanco-amarillentos.

El examen histológico (Fig. 5) mostraba en todos los nódulos, proliferación de células fusiformes, constituyendo haces irregulares y formando palizadas nucleares. Algunos elementos nucleares eran grandes e hipercromáticos, sin actividad mitótica. En algunas áreas el estroma es laxo y de mayor celularidad. Destacaban algunos 


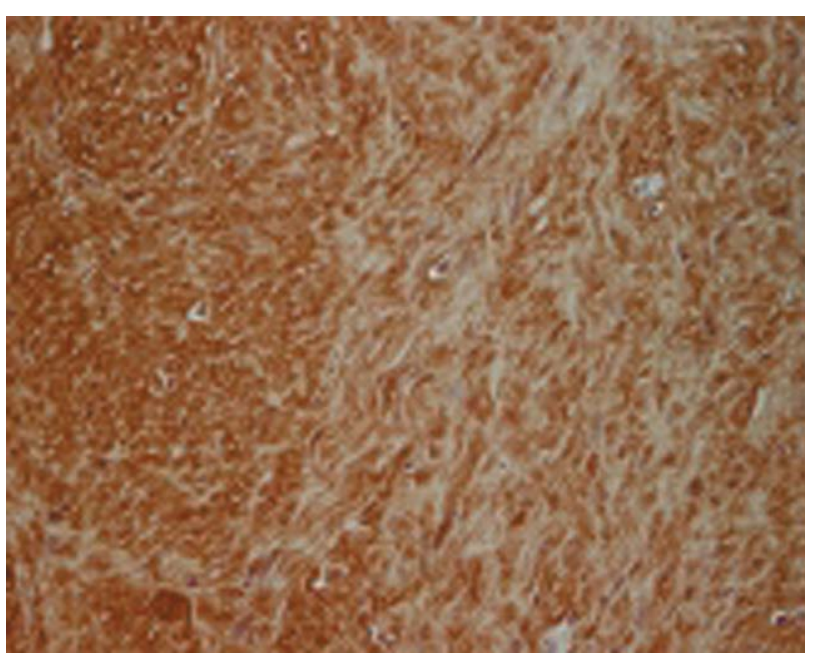

FIGURA 5. Examen citoquímico demostrando la marcación fuerte para la proteina S-100 (PS-100 40x).

vasos de pared engrosada; todos los nódulos son revestidos por haces fibrosos. El estudio inmunocitoquímico era negativo para desmina, HHF35 (anticuerpo específico para la actina muscular) y CD34 (antígeno de pared de las células endoteliales y células stem hematopoyéticas) y positivo para vimentina $\mathrm{E}$ y especialmente para proteína PS-100 (PS-100).

Por lo tanto el diagnóstico definitivo fue de schwanoma benigno múltiple del pene.

Nueve meses después de la exéresis quirúrgica no existía recidiva.

\section{DISCUSIÓN}

Verocay en 1910 fue quien describió por primera vez un tumor que se desarrolla en la vainas nerviosas. Masson en 1932 demuestra que este tumor derivaba de las células de Schwan, y pasó a llamarse schwanoma ${ }^{5}$. Peral Aranda en 1954 realiza la primera descripción de este tumor en el pene $^{6}$.

En 1996 Hamilton, et al. describen el primer caso de schwanoma múltiple del pene ${ }^{7}$.

La última revisión de este tipo de tumores fue realizada en 2001 por Cátedra García, et al. ${ }^{6}$ identificando 19 casos.

En este momento se contabilizan 28 descripciones (con el caso presente) en la literatura (Tabla 1). La mayoría de los schwanomas eran únicos $(\mathrm{n}=21 / 72 \%)$, de 0,4 a $8 \mathrm{~cm}$ de diámetro, localizados en la cara dorsal del pene $(n=14 /$ $50 \%$ ) y la edad media de los pacientes fue de 36,0 años (2-78); los enfermos con schwanomas múltiples $(n=7 / 25 \%)$ presentaban una edad media ligeramente superior (40 años). Está descrita una recidiva tardía (7 años) de un schwanoma solitario benigno (Tabla 1 , caso 3 ).

Algunas formas de schwanoma tienen degeneración maligna y una asociación elevada con la enfermedad de Von Recklinghausen ${ }^{8}$. En la revisión de Cátedra García ${ }^{6}$, todas las formas malignas $(n=3 / 12 \%)$ estaban asociadas a esta enfermedad. La degeneración maligna de un schwanoma benigno (en cualquier localización) se calcula en 1/1000 según Enzinger e Weiss ${ }^{9}$.

No se encuentran identificados factores etiopatogénicos para su desarrollo, aunque se ha asociado a la pérdida o a la alteración del gen de la neurofibromatosis tipo II $^{2}$.

Los síntomas clínicos dependen de la localización y del volumen de la lesión, en el pene dependerá del grado de deformación y de la asimetría peneana que llevan al paciente a la consulta. Al examen físico, estas lesiones se presentan como nódulos subcutáneos únicos o múltiples, de consistencia duro-elástica, de localización variable en el pene y no se encuentran adherentes a los planos superficiales ni profundos.

Mediante el estudio ecográfico aparecen formaciones quísticas de ecogenicidad variable localizadas superiormente a la túnica albuginea cavernosa. La tomografia axial computerizada y la resonancia magnética nuclear ayudan a localizar y delimitar las lesiones.

El diagnóstico diferencial se realiza con los tumores subcutáneos que pueden aparecer en el pene: ateroma, lipoma, dermatofibroma, neurofibroma, leiomiosarcoma, enfermedad de La Peyronie y granuloma de cuerpo extraño ${ }^{4,10}$.

El tratamiento consiste en la exéresis quirúrgica completa, habitualmente con denudación peniano y circuncisión, debido a que la localización más frecuente es en el cuerpo del pene.

El diagnóstico definitivo es dado por el examen histológico de las lesiones.

Histológicamente se diferencian dos tipos de disposición celular: el tipo A de Antoni de estructura densa, ricamente celular, con núcleos dispuestos en empalizada- los cuerpos de Verocay; y 
Tabla 1

Revisión de casos

\begin{tabular}{|c|c|c|c|c|c|c|c|c|}
\hline Caso & Año & Autor & Diagnóstico & Edad & Localización & Número & Volúmen & Recidiva \\
\hline $1^{6}$ & 1954 & Peral & Neurinoma & 35 & Dorso & Único & $8 \mathrm{~cm}$ & No \\
\hline $2^{6}$ & 1968 & Parra & Neurinoma & 28 & Glande & Único & $\mathrm{Nd}$ & $\mathrm{Nd}$ \\
\hline $3^{6,8,9}$ & 1970 & Dehner & Neurilemoma & 24 & Prepúcio & Único & $1,5 \mathrm{~cm}$ & $\mathrm{Si}$ (7 años) \\
\hline $4^{6,8,9}$ & 1970 & Dehner & Neurilemoma & 36 & Dorso & Único & $1,5 \mathrm{~cm}$ & No \\
\hline $5^{6,8,9}$ & 1970 & Dehner & Neurilemoma & 25 & Dorso & Único & $4 \mathrm{~cm}$ & No \\
\hline $6^{6,8,9}$ & 1970 & Dehner & $\begin{array}{l}\text { Schwanoma } \\
\text { maligno }^{\mathrm{a}}\end{array}$ & 15 & $\begin{array}{l}\text { Prepúcio, } \\
\text { glande }\end{array}$ & Único & $4 \mathrm{~cm}$ & $\mathrm{Nd}$ \\
\hline $7^{6,8,9}$ & 1970 & Dehner & $\begin{array}{l}\text { Schwanoma } \\
\text { maligno }^{\mathrm{a}}\end{array}$ & 2 & Dorso & Único & $2,5 \mathrm{~cm}$ & No \\
\hline $8^{6,8,9}$ & 1974 & Fethiere & $\begin{array}{l}\text { Schwanoma } \\
\text { plexiforme }\end{array}$ & 16 & Dorso & Único & $8 \mathrm{~cm}$ & No (2 años) \\
\hline $9^{3,6,8,9}$ & 1980 & Marsidi & Schwanoma & 24 & Dorso & Múltiple & $1 \mathrm{e} 0,5 \mathrm{~cm}$ & No \\
\hline $10^{6,8,9}$ & 1984 & Nishio & Schwanoma & 19 & Ventral & Único & $1 \mathrm{~cm}$ & $\mathrm{Nd}$ \\
\hline $11^{8}$ & 1988 & Fujito & & 34 & Prepúcio & Único & $\mathrm{Nd}$ & $\mathrm{Nd}$ \\
\hline $12^{6,8}$ & 1989 & Mohanty & Schwanoma & 30 & Glande & Único & $1 \mathrm{~cm}$ & $\mathrm{Nd}$ \\
\hline $13^{5,6,8}$ & 1990 & Chan & Neurilemoma & 30 & Freno & Único & $2,5 \mathrm{~cm}$ & No \\
\hline $14^{8}$ & 1990 & Watanabe & Schwanoma & 78 & Dorso & Múltiple & $\mathrm{Nd}$ & $\mathrm{Nd}$ \\
\hline $15^{8}$ & 1992 & Kawabata & Schwanoma & 20 & Prepúcio & Único & $\mathrm{Nd}$ & $\mathrm{Nd}$ \\
\hline $16^{6,8,9}$ & 1993 & Kubota & Schwanoma & 74 & Dorso & Único & $2 \mathrm{~cm}$ & No \\
\hline $17^{6,8}$ & 1995 & Aloi & $\begin{array}{l}\text { Schwanoma } \\
\text { maligno }^{a}\end{array}$ & 58 & Glande & Único & $\mathrm{Nd}$ & $\mathrm{Nd}$ \\
\hline $18^{1,6,8}$ & 1995 & Mayersak & Schwanoma & 53 & Dorso & Único & $0,6 \mathrm{~cm}$ & No \\
\hline $19^{6,7,8}$ & 1996 & Hamilton & Neurilemoma & 23 & Lateral & Múltiple (3) & $\mathrm{Nd}$ & $\mathrm{Nd}$ \\
\hline $20^{6,9}$ & 1998 & Berard & Schwanoma & 64 & $\begin{array}{l}\text { Surco balano- } \\
\text { Prepucial }\end{array}$ & Único & $\mathrm{Nd}$ & $\mathrm{Nd}$ \\
\hline $21^{6,8}$ & 1998 & Suzuki & Schwanoma & 65 & Dorso & Múltiple & $\mathrm{Nd}$ & $\mathrm{Nd}$ \\
\hline $22^{8}$ & 1999 & Sato & Schwanoma & 65 & Dorso & Múltiple (5) & $3,5 \mathrm{~cm}$ (mayor) & No (6 meses) \\
\hline $23^{6}$ & 1999 & Garcia & Schwanoma & 28 & Dorso & Único & $1,2 \mathrm{~cm}$ & No (18 meses) \\
\hline $24^{11}$ & 2000 & Pandit & Neurilemoma & 10 & Dorso & Múltiple (4) & 0,5 a $1,2 \mathrm{~cm}$ & No (6 meses) \\
\hline $25^{4}$ & 2003 & Amara & Schwanoma & 34 & Dorso & Único & $1,5 \mathrm{~cm}$ & No (18 meses) \\
\hline $26^{2}$ & 2003 & Kiang & Schwanoma & 28 & $\begin{array}{l}\text { Surco balano- } \\
\text { prepucial }\end{array}$ & Único & $2 \mathrm{~cm}$ & No (6 años) \\
\hline $27^{2}$ & 2003 & Jiang & Schwanoma & 34 & Lateral & Único & $0,5 \mathrm{~cm}$ & No (3 años) \\
\hline 28 & 2005 & $\begin{array}{l}\text { Caso } \\
\text { presente }\end{array}$ & Schwanoma & 57 & Lateral & Múltiple (4) & 0,4 a $2 \mathrm{~cm}$ & No (9 meses) \\
\hline
\end{tabular}

${ }^{a}$ Asociados a la enfermedad de Von Recklinghausen. Nd- sin datos disponibles.

el tipo B de Antoni de estructura laxa y edematosa, de menor celularidad y estroma mixoide ${ }^{9,10}$; frecuentemente se encuentran los dos padrones celulares en la misma lesión. No se encuentra definida la asociación entre cualquier de estos tipos y la probabilidad de recidiva o degeneración a malignidad.
Las células exprimen la proteína PS-100 que es primordial para el diagnóstico, y no exprime la enolasa neuronal específica (ENE) ni a los marcadores de los neurofilamentos. La PS-100 puede aparecer en tumores derivados de las vainas de Schwan (schwanoma, neurofibroma e neurinoma) y de los melanocitos (nevus y melanoma) ${ }^{8}$. 
La recidiva de este tipo de tumores no se espera, sin embargo exigen vigilancia clínica, debido a que pueden sufrir transformación maligna ${ }^{2}$.

\section{CONCLUSIÓN}

Los schwanomas solitarios parecen no tener cualquier significado particular, sin embargo los múltiples se deben considerar marcadores de una afección sistémica. Los tumores que son malignos frecuentemente se asocian a la enfermedad de Von Recklinghausen (neurofibromatosis tipo 1).

Con todo su localización en el pene es rara.

\section{REFERENCIAS}

1. Mayersak JS, Viviano CJ, Babiarz JW. Schwannoma of the penis. J Urol. 1995;153(6):1931-1932.

2. Jiang R, Chen JH, Chen M, Li QM. Male genital Schwannoma, review of 5 cases. Asian J Androl. 2003 sep;5(3):251254 .

3. Marsidi PJ, Winter CC. Schwannoma of the penis. Urology. 1980 sep; 16(3):303-304.

4. Amara N, Leroy X, Nivet J, Biserte J, Mazeman E. Schwannome du pénis: une tumeur rare et inhabituelle des organes génitaux externes. Prog Urol. 2003;13(2):324-325.
5. Chan WP, Chiang SS, Huang AH, Lin CN. Penile frenulum neurilemoma: a rare and unusual genitourinary tract tumor. J Urol. 1990;144(1):136-137.

6. Cátedra García A, Sanz Jaka JP, Garmendia Olaizola G, Arrinda Yeregui JM, Aranzadi F. Arch Esp Urol. 2001;54 (7):713-715.

7. Hamilton DL, Dare AJ, Chilton CP. Multiple neurilemmomas of the penis. Br J Urol. 1996;78(3):468-469.

8. Kubota Y, Nakada T, Yaguchi H, Abe Y, Sasagawa I. Urol Int. 1993;51(2):111-113.

9. Berard F, Grézard P, Ruffion N, Balme B, Perrot H. Schwannome solitaire du sillon balano-préputial. Ann Dermatol Venereol. 1998;125(10):729-731.

10. Sato D, Kase T, Tajima M, Sawamura Y, Matsushima M, Wakayama M, et al. Penile Schwannoma. Int J Urol. 2001; 8(2):87-88.

11. Pandit SK, Rattan KN, Gupta U, Budhiraja S, Singh Y, Sen $\mathrm{J}$, et al. Multiple neurilemmomas of the penis. Pediatr Surg Int. 2000; 16 (5-6):457.

Dr. R. Simeão Versos

E-mail: ruiversos@yahoo.com.br;

(Trabajo recibido el 13 de septiembre 2005) 